\title{
EARTH OBSERVATION TO SUBSTANTIATE THE SUSTAINABLE DEVELOPMENT GOAL 11: PRACTICAL CONSIDERATIONS AND EXPERIENCES FROM AUSTRIA
}

\author{
T. Blaschke ${ }^{1, *}$, A. Kovács-Győri ${ }^{1}$ \\ ${ }^{1}$ Dept. of Geoinformatics, University of Salzburg, Schillerstr. 30, 5020 Salzburg, Austria (thomas.blaschke, anna.gyori)@sbg.ac.at
}

Inter-Commission WG III/IVb

KEY WORDS: Sustainable Development Goals, Earth Observation, Urban Green, Well-being, Quality of Life

\begin{abstract}
The 2030 Agenda for Sustainable Development is widely appreciated and increasingly known by a wider public. However, less obvious are the enormous coordination and harmonization efforts to reify these goals into 169 targets and 232 indicators. We exemplarily outline a tangible pathway to address SDG11 and one associated indicator 11.7.1 "Average share of the built-up area of cities that is open space for public use for all, by sex, age and persons with disabilities". We highlight some specific problems for reporting on indicators related to urban green spaces (UGS) and make suggestions for this indicator by illustrating the potential of Earth Observation data and spatial accessibility analysis.
\end{abstract}

\section{EARTH OBSERVATION AND THE SUSTAINABLE DEVELOPMENT GOALS}

The 2030 Agenda for Sustainable Development was adopted by the United Nations General Assembly in September 2015. With 17 Sustainable Development Goals (SDGs), 169 targets, and a global indicator framework, consisting of 232 indicators, the 2030 Agenda requires to consider new and innovative means to restrain the many development challenges in order to 'leave no one behind'. The SDGs address processes that occur over a wide range of spatial and temporal scales, from abrupt changes such as earthquakes to decadal shifts such as growth and shrinkage of ice sheets. As the implementation of the 2030 Agenda gains momentum, Member States and the global community have begun to utilize geospatial information and Earth observation (EO) information as fundamental inputs for realizing the 2030 Agenda.

Organisations such as the Group on Earth Observations (GEO), the Committee on Earth Observations Satellites (CEOS), the United Nations Committee of Experts on Global Geospatial Information Management (UN-GGIM) or the World Bank have undertaken significant effort to support lower to middle-income countries. They provide a basis and guidance when developing and strengthening their national and sub-national arrangements in geospatial information management and related infrastructures. UN-GGIM sets the directions for the production and use of geospatial information within national and global policy frameworks and for building and strengthening geospatial information capacity of nations, especially of developing countries. This partnership of UN organisations and organisations such as the World Bank, GEO, CEOS, and other global actors, aims to make the SDGs, their targets and indicators concrete and tangible, in fact reportable for Member States.

EO data - including satellite-borne, airborne, or drone-based imagery - have almost arrived at "everybody's desktop" - at least day-to-day users appreciate the bird's eye view of Google
Earth on similar applications. While EO helps scientists to understand landscapes, weather and climate conditions or support the forecast of crop yields, EO data can also be used for specific and tangible reporting of the status quo of various environmental conditions.

In 2018, CEOS published a handbook (European Space Agency (ESA), 2018) that analyses the potential role of EO for the SDGs, their targets and indicators in a remarkably tangible way. The handbook provides valuable guidance and captures the different dimensions and perspectives on the role of EO data, including specific examples, for supporting the implementation of the SDGs. This handbook informs National Statistical Offices and the broader community as to how geospatial information, Earth observation and other data sources can reliably and consistently contribute to the 2030 Agenda for Sustainable Development.

For the remainder of the article we build on the global Integrated Geospatial Information Framework (GIF). In particular, we will exemplarily outline a tangible pathway to address SDG11 and one of the associated indicators. For indicator 11.7.1 "Average share of the built-up area of cities that is open space for public use for all, by sex, age and persons with disabilities", we will highlight some specific problems for reporting on indicators related to urban green spaces (UGS). In the practical part, we will make suggestions for this indicator and report on attempts from an Austrian-wide initiative called UniNetZ where scientists collaborate to support national agencies with concrete advice for operationalizing the regular reporting of the status of the indicators.

\section{SDG 11.7 - HOW TO ASSESS URBAN GREEN ACCESSIBLITY EFFECTIVELY?}

\subsection{The Role of UGS in Cities}

UGS can enhance people's well-being in cities as areas for recreation and exercise or simply as an antithesis to the rush of

* Corresponding author 
urban life. UGSs directly experienced or visually enjoyed, significantly increases the attractiveness of urban areas and the quality of life therein. This is particularly applicable for small UGSs (pocket parks) that can be relished for the moment and contribute to restorative effects in a residential environment. Increasing traffic congestion, ever-present noise, and widespread, ongoing construction diminish free space and necessitate the maintenance of green spaces and the creation of new ones. Ongoing discussions in politics, media, and the public demonstrate the importance of this topic.

As UGS is an integrative concept, it is usually not enough (although better than doing nothing) to install single green elements in a pedestrian zone or planting a single tree in front of a skyscraper. UGS comprises several aspects: (1) urban ecosystems and biodiversity, manifested in the physical green (i.e., vegetative environment); (2) the psychological well-being and QoL, including restorative effects and recovery of attention induced by green structures and non-monotonous urban landscapes; and (3) green mobility and production and consumption including resource maintenance and efficiency in the sense of the green city (Lang et al., 2018).

Within the SDG framework, UGS is only one aspect out of many aspects that are believed to contribute to a good life. UGS is inherent in several sustainability aspects and is particularly reflected in SDG goal 11.7: "By 2030, provide universal access to safe, inclusive and accessible, green and public spaces, in particular for women and children, older persons and persons with disabilities". SDG goal 11.7 is almost directly translated to indicator 11.7.1 "Average share of the built-up area of cities that is open space for public use for all, by sex, age, and persons with disabilities" (1).

This indicator - again, this is just one out of 232 - is understandable to the broader public but its implementation is challenging, particularly if it shall support its quantification and reporting in a standardized or even automated fashion. In fact, the devil is in the details: While a UGS layer can be developed relatively straightforwardly, as a kind of baseline product by means of remote sensing, its counterpart "public space" is more challenging since it does not refer to a land cover but a land use category. Even more difficult is the translation of the term "accessible" into reportable procedures. To reify such accessibility, a reporting agency needs EO data but auxiliary data such as authoritative GIS data layers or data like OpenStreetMap and, in fact, a 'clever' combination. We will report on first attempts to develop solutions for several major cities in Austria with inhabitant numbers ranging from 1.9 Million (Vienna) to Salzburg (155.000).

\subsection{Conceptual and Methodological Limitations in Analysing UGS Access}

Figure 1 shows the main conceptual and methodological questions that are crucial for a reliable UGS accessibility analysis. Based on existing literature we tried to summarize consensus and limitations.

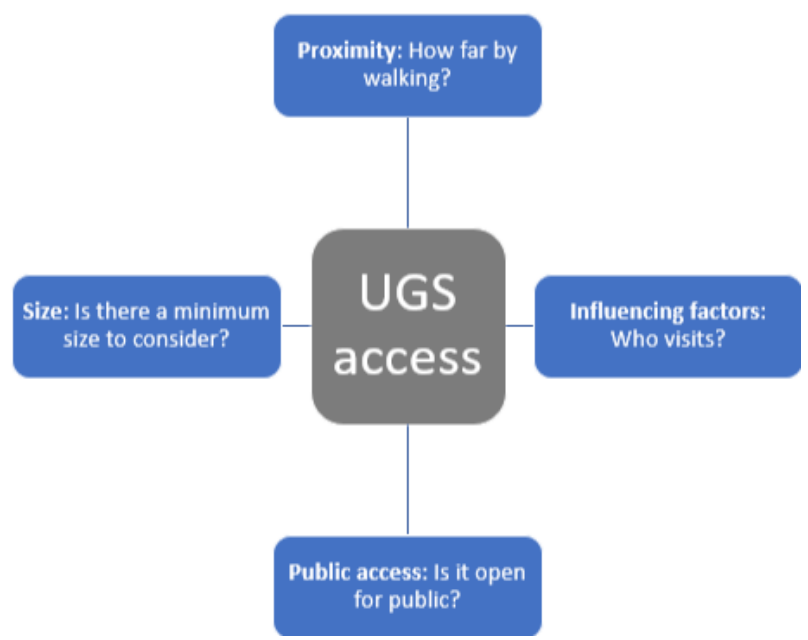

Figure 1. Conceptual and methodological questions in UGS accessibility analysis

2.2.1 Proximity: It has been proven in various studies that nearby green space supports human health and that proximity to UGS matters (Chiesura, 2004; Ekkel and de Vries, 2017; Hartig and Kahn, 2016; Kothencz and Blaschke, 2017; Kovacs-Györi et al., 2018; Lee and Maheswaran, 2011; Sturm and Cohen, 2014; Wolch et al., 2014; Wood et al., 2017). However, there is little consensus on what "nearby" means or how big this ideal distance to UGS should be. The SDG 11.7.1 indicator refers to the "average share" of open public spaces (United Nations General Assembly, 2015), which is a relatively easily extractable statistic for a city. However, it does not necessarily reveal the "real" accessibility of UGS, especially not on finer spatial scales. For example, the WHO suggests around $50 \mathrm{~m}^{2}$ of UGS per capita in cities as an ideal amount (WHO, 2012). Such an average value for a whole city it is not able to reveal differences in the spatial distribution or real proximity to given residents. Figure 2 illustrates this by two scenarios where the area (grey), the population and the extent of UGS (dark green) of a city are equal in both cases, but the distribution is different. It results in larger areas covered by ideal accessibility (light green) but also a shorter maximum distance to the closest park. However, as it is detailed below, variety in UGS sizes might mean different levels of hierarchy with different functions and frequency of visit. Therefore, although many smaller UGSs might provide a better accessibility, they do not substitute larger parks due to differences in their characteristics.
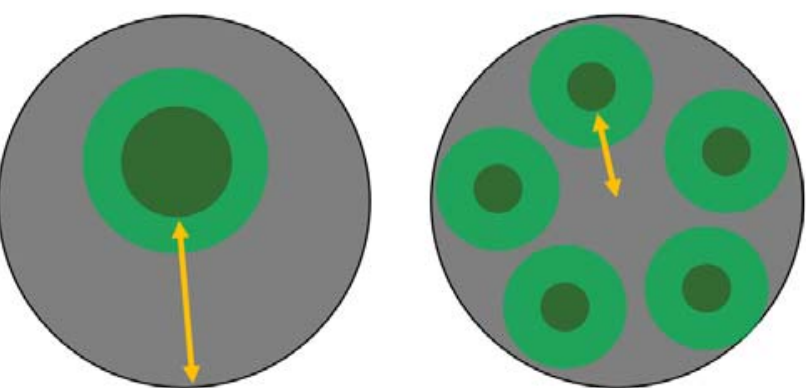

Figure 2. Illustration for the importance of accessibility and proximity beyond the amount of UGS (dark green - UGS, light green - ideal walking distance, yellow arrow - maximum walking distance to the closest UGS)

(1) 11.7.2 is non-spatial: „Proportion of persons victim of physical or sexual harrasment" 
An often-suggested ideal distance to UGS is a 5-minute walk (usually equal to $300-500 \mathrm{~m}-300$ as crow flies, 500 on foot (Tarzia, 2003)) to the closest, usually smaller (e.g. 1 ha) green space (Ekkel and de Vries, 2017; Gupta et al., 2016; Handley, 2003; Russo and Cirella, 2018). This approach considers spatial relations. However, as Gupta et al., (2016) pointed out: even "300-400 m can be high for children due to increased motorized traffic and parent's anxiety for security of their wards" (p. 200). Furthermore, perceived distances, especially perceived safety is also crucial for women - another prioritized group in SDG 11.7. This means that accessibility cannot be reduced to objective numbers only, when aiming to enhancing the overall urban quality of life. Interestingly, when considering positive health effects, the extensive literature review Ekkel and de Vries reveals that "using larger distances as cut-off point sometimes results in stronger associations between access to green space and health parameters than using shorter distances" (Ekkel and de Vries, 2017, p. 217). We may conclude that a universal UGS accessibility threshold may be insufficient. Likewise, the positive effect of other types of green such as plants on the balconies or courtyard gardens, should not be neglected either (Ekkel and de Vries, 2017; Hartig and Kahn, 2016; Russo and Cirella, 2018) although its implementation is expensive and ambiguous due to the very small sizes and manifestations.

2.2.2 Influencing factors: Measuring the walking distance is a more detailed and expedient way to consider the local inequalities of access (e.g. Comber et al., 2008; Gupta et al., 2016). However, also walking distance as a proxy is prone to strong generalizations of the parameters that may explain park visits, These parameters include sense of security, social encounters, health condition, social status, or age of the visitor (Hartig et al., 2014; Kothencz and Blaschke, 2017; Lindberg and Schipperijn, 2015; Ode Sang et al., 2016; Schetke et al., 2016; Wang et al., 2015). Walking distance analysis is a straightforward GIS-based method but may not account for all planning measures that aim to enhance the well-being of urban residents, or understand the real phenomenon behind "providing safe, inclusive and accessible green spaces" to different social groups. As our approach is currently in its initial phase, we will emphasize the potential of EO and GIS mainly for the former category (walking distance to UGS) but we draw attention to further necessary steps and potential limitation in order to achieve SDG 11.7.

2.2.3 Public access and size: For the sake of simplicity, the third and fourth dimensions outlined in Figure 1 are summarized in this sub-section. We already mentioned that UGS can be straightforwardly extracted from EO data, but the procedure should be standardized. Data sources and analysis steps need to transparent and transferable to produce comparable results on national and international levels. Unfortunately, we cannot extract public access directly from satellite or airborne images. This causes limitations to the analysis and poses the challenge of finding adequate data sources to cross-validate whether a place is publicly available or not.

Based on the conclusions of Ekkel and de Vries (2017) we should consider UGS sizes and their large variations. By applying increasing minimum distances from home to different UGS hierarchy levels (see more details in sections 2.3 and 3), we can get a better picture of the overall perceived greenness even if not all of this green is publicly accessible. Most of these areas are visible and therefore have a certain effect - even if such a situation is different from an actual park visit. Excluding
UGS merely based on their size or the lacking access might distort the picture because some of its advantages are still present such as aesthetic, mitigating urban heat or air pollution functions.

\subsection{Existing approaches}

Ekkel and de Vries (2017) identified two main types of metrics for UGS access: residential proximity accessibility metrics vs. cumulative opportunity metrics (Figure 3). For the first category, they identified two sub-categories: 1) distance to the nearest; 2) whether or not a qualifying green area is available within a given distance. Whereas in the latter group (cumulative), there area approaches calculating within an administrative area, or within a certain distance from home.

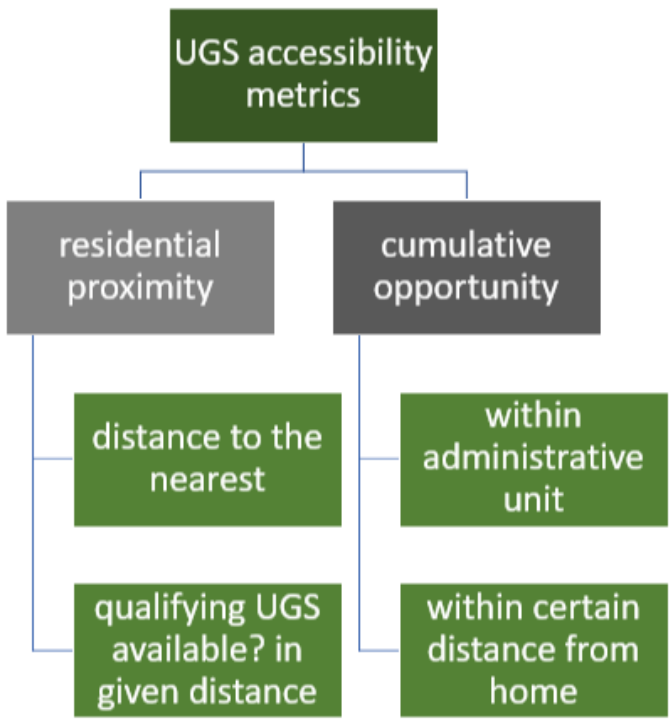

Figure 3. Categorization of UGS accessibility metrics (based on Ekkel and de Vries 2017)

Cumulative metrics are important, as not only the nearest green space matters but more nearby spaces offer more possible contacts and other benefits. Moreover, considering more than just the nearest UGS usually means that these green spaces are diverse, and therefore in its total more likely to satisfy the expectations of different residents. These different needs and expectations (also for the same person over time) are also reflected in the concept of "recreation opportunity spectrum" (e.g. Gundersen et al., 2015).

UGS accessibility studies often use street network data and calculate their distances in walking time; however, they rarely use remote sensing images as a starting point to extract UGS. Gupta et al. (2016) proposed a methodology using a cumulative approach and remote sensing images. They distinguished UGS at different urban hierarchical levels from tot lots to urban forests and analysed their accessibility using network distances of pedestrians. We believe that this approach can handle most of the mentioned limitations in Section 2, by considering variance in UGS and real accessibility to the best possible degree. According to Ekkel and de Vries (2017), our proposed approach falls into the cumulative category. This category tends to provide results that are more reliable. Instead of administrative units or distances from each individual building, we defined catchment areas from UGS entry points to facilitate easier comparisons between cities or given areas without the 
need for an additional data layer containing residential buildings.

\section{PROPOSED WORKFLOW FOR AUSTRIA}

\subsection{Goals}

Our goal in developing the workflow is to develop a transferable methodology for extracting UGS from EO data as an input for an accessibility analysis, considering different levels of UGS hierarchy. With underlying socio-economic data, this approach can be used to map the access to UGS according to the SDG 11.7.1 indicator. The current work does not investigate follow-up steps, such as planning actions that aim to provide better access for selected groups. Still, the results of our approach shall support these endeavours - also for stakeholders who are not familiar with GIS technology or EO. In our approach, we intend to extract UGS from satellite images, using OBIA instead of pixel-based classification. If available, highresolution land use data can enrich the analysis by excluding areas that are not publicly available, but as mentioned in 2.2.3, public access only makes a difference in actual park visits. As discussed, there are several other aspects of UGS for residents.

\subsection{Input data}

For the Austrian case study, we use the following data sets.

1. EO data: Sentinel-2 with spatial resolution of $10 \mathrm{~m}$ for July 2019

2. Open Street Map (OSM) to extract street networks for the selected city. This street network will be used to calculate walking distances to UGS.

3. (optional) For European cities having at least 100.000 inhabitants, the Urban Atlas data set is available as a detailed vector-based land use map, provided by the European Environmental Agency. This can be used to exclude private areas extracted as UGS from EO data.

4. (optional) To fully reflect SDG 11.7.1, we also need socio-economic data to define the UGS access for different age and gender groups, ideally also for handicapped persons as a separate category. In the case of Austria, this dataset is available at a $100 \mathrm{~m}$ resolution provided by Statistics Austria. Without this information, the results of the accessibility analysis still provide a good approximation of the areas within the city with poorer UGS access.

All the remaining parameters and metrics, such as the entry points of UGS to measure the distance to will be derived from these data sets.

\subsection{Analysis steps}

Considering also the limitations mentioned in 2.2, we suggest a workflow (Figure 4):

1. The first step includes satellite data acquisition and the extraction of UGS patches using an object-based approach. For Europe, the satellite images should reflect the conditions of a summer month and high vegetation biomass.

2. After the UGSs are extracted and their sized calculated, thresholds will be applied to remove outliers.

3. UGSs will be clustered - instead of a fixed UGS hierarchy categorization based on the size. The smallest ones will be considered as residential, followed by neighbourhood, quarter, district and city green, having urban forest as the biggest category (Gupta et al., 2016). This way we can provide a more "fuzzy" interpretation of the UGS sizes.

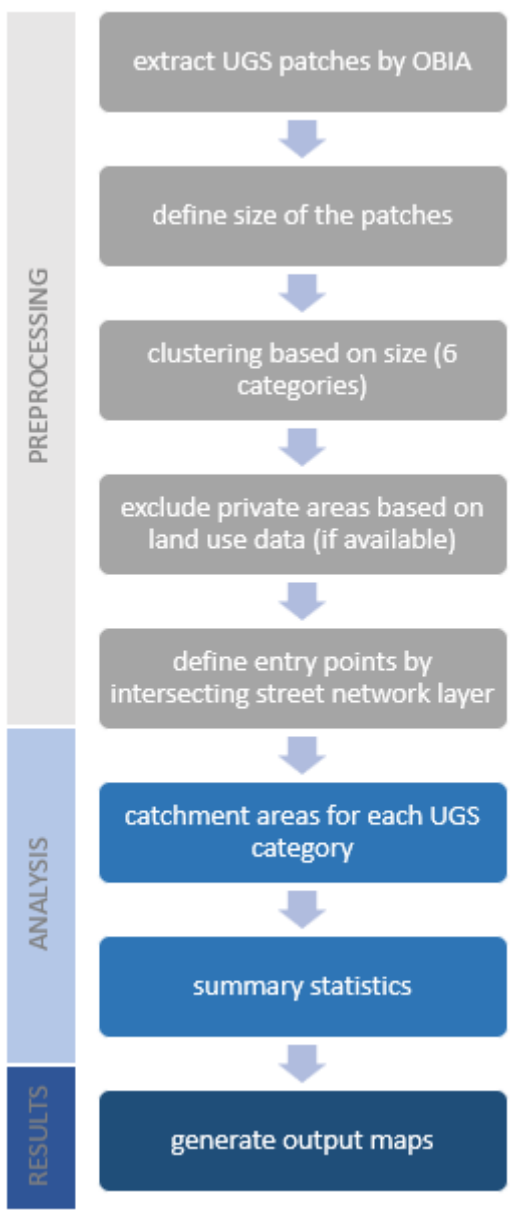

Figure 4. Proposed workflow to analyse UGS accessibility using EO data and OSM street network

4. If reliable land use data is available, UGSs that are not publicly available will be extracted.

5. Once the street network from OSM is extracted adequately (facilitating accessibility analysis), we can define the entry points of the UGSs by overlaying the street network and UGS layer to identify where pathways intersect with the outline of a UGS (using a $25-50 \mathrm{~m}$ threshold to reduce the error originating from spatial inaccuracies of the data).

6. The next step is to define the catchment areas of the different UGS clusters in terms of walking distances. Based on Gupta et al. 2016 and by adding a 10\% fuzziness buffer - a maximum of $165 \mathrm{~m}$ for residential green, $440 \mathrm{~m}$ for neighbourhood green, $880 \mathrm{~m}$ for quarter green, $1760 \mathrm{~m}$ for district green, $3520 \mathrm{~m}$ for city green, and $5500 \mathrm{~m}$ for urban forests is applied, respectively.

7. Once general walking accessibilities are calculated, the next step is to provide the interpretation for the results combined with the available socio-economic information. On city scale, areas with poor UGS access (of any category) can be identified. Whereas considering the cells of the socio-economic data, we 
can define whether people living in that $100 \times 100 \mathrm{~m}$ area have adequate access to different kinds of UGS.

8. The last step is generating the output maps of the results.

If the practical implementation for major Austrian cities reveals data access problems, one alternative option for an NDVI-based approach is described by (Ekkel and de Vries, 2017). This approach does not extract UGS patches. NDVI values could be calculated for given analysis units defined by the socioeconomic data, e.g. $100 \times 100 \mathrm{~m}$ cells in the case of Austria. The results of this approach can be used on its own as a basic overview for a city or as complementary statistics to the described workflow. From a planning perspective, it is especially important to increase the amount of green where the general greenness (NDVI) is lower (Keul et al., 2017). This is because areas with low or no amount of green can profit even from planting a few trees, whereas the same action would make almost no difference in another area that is considered green already due to the non-linear characteristic of the positive effect of urban green.

\section{CONCLUSIONS}

To conclude, it is important to emphasize that indicator measures of the SDG 11.7 require an approach that is able to handle the complex nature of UGS. This include among others the precise definition of accessibility per se, along with considering UGS at different levels of hierarchy by not limiting based on their size to better represent the behaviour of urban dwellers in terms of their needs and perceptions regarding UGS. Methods that are planned to be used to substantiate SDG goals related to urban phenomenon should rely on data sets that are widely available to provide a transferable solution and support comparisons on national and international scales. Therefore, we proposed a workflow based on EO data, OSM street network, and GIS-based accessibility calculations to support the endeavours of SDG 11.7. At the same time, we also highlighted the most common conceptual and methodological limitations to reveal the gap between existing indicators and the envisioned goals of accessible UGS.

\section{REFERENCES}

Chiesura, A., 2004. The role of urban parks for the sustainable city. Landsc. Urban Plan. https://doi.org/10.1016/j.landurbplan.2003.08.003

Comber, A., Brunsdon, C., Green, E., 2008. Using a GIS-based network analysis to determine urban greenspace accessibility for different ethnic and religious groups. Landsc. Urban Plan. https://doi.org/10.1016/j.landurbplan.2008.01.002

Ekkel, E.D., de Vries, S., 2017. Nearby green space and human health: Evaluating accessibility metrics. Landsc. Urban Plan. 157 , 214-220.

https://doi.org/10.1016/j.landurbplan.2016.06.008

European Space Agency (ESA), 2018. Satellite Earth Observations in support of the Sustainable Development Goals, The CEOS Earth Observation Handbook. Special 2018 Edition.

Gundersen, V., Tangeland, T., Kaltenborn, B.P., 2015. Planning for recreation along the opportunity spectrum: The case of Oslo, Norway. Urban For. Urban Green.
https://doi.org/10.1016/j.ufug.2015.01.006

Gupta, K., Roy, A., Luthra, K., Maithani, S., Mahavir, 2016. GIS based analysis for assessing the accessibility at hierarchical levels of urban green spaces. Urban For. Urban Green. 18, 198-211. https://doi.org/10.1016/j.ufug.2016.06.005

Handley, J., 2003. Accesible natural green space. Standards in towns and cities: A review and toolkit for their implementation. English National Research Reports. http://publications.naturalengland.org.uk/publication/65021

Hartig, T., Kahn, P.H., 2016. Living in cities, naturally. Science (80-. ). https://doi.org/10.1126/science.aaf3759

Hartig, T., Mitchell, R., de Vries, S., Frumkin, H., 2014. Nature and Health. Annu. Rev. Public Health. https://doi.org/10.1146/annurev-publhealth-032013-182443

Keul, A.G., Brunner, B., Blaschke, T., 2017. Urban Quality of Life: A Rubik cube of objective and subjective descriptors. Momentum Quaterly. Zeitschrift für Sozialen Fortschritt 6, $123-137$.

Kothencz, G., Blaschke, T., 2017. Urban parks: Visitors' perceptions versus spatial indicators. Land Use Policy. https://doi.org/10.1016/j.landusepol.2017.02.012

Kovacs-Györi, A., Ristea, A., Kolcsar, R., Resch, B., Crivellari, A., Blaschke, T., 2018. Beyond Spatial Proximity Classifying Parks and Their Visitors in London Based on Spatiotemporal and Sentiment Analysis of Twitter Data. ISPRS $\begin{array}{lllll}\text { Int. J. } & \text { Geo-Information } & 7,\end{array}$ https://doi.org/10.3390/ijgi7090378

Lang, S., Blaschke, T., Kothencz, G., Hölbling, D., 2018. Urban green mapping and valuation, in: Urban Remote Sensing, Second Edition. https://doi.org/10.1201/9781138586642

Lee, A.C.K., Maheswaran, R., 2011. The health benefits of urban green spaces: a review of the evidence. J. Public Health (Bangkok). https://doi.org/10.1093/pubmed/fdq068

Lindberg, M., Schipperijn, J., 2015. Active use of urban park facilities - Expectations versus reality. Urban For. Urban Green. 14, 909-918. https://doi.org/10.1016/j.ufug.2015.08.007

Ode Sang, Å., Knez, I., Gunnarsson, B., Hedblom, M., 2016. The effects of naturalness, gender, and age on how urban green space is perceived and used. Urban For. Urban Green. 18, 268-276. https://doi.org/10.1016/j.ufug.2016.06.008

Russo, A., Cirella, G.T., 2018. Modern compact cities: How much greenery do we need? Int. J. Environ. Res. Public Health. https://doi.org/10.3390/ijerph15102180

Schetke, S., Qureshi, S., Lautenbach, S., Kabisch, N., 2016. What determines the use of urban green spaces in highly urbanized areas? - Examples from two fast growing Asian cities. Urban For. Urban Green. https://doi.org/10.1016/j.ufug.2016.02.009

Sturm, R., Cohen, D., 2014. Proximity to urban parks and mental health. J. Ment. Health Policy Econ. https://doi.org/10.1016/j.biotechadv.2011.08.021.Secreted 
Tarzia, V., 2003. European Common Indicators - Towards a Local Sustainability Profile, Luxemburg, European Commission. $\quad$ https://op.europa.eu/en/publication-detail//publication/83090cd3-b9b2-4d81-9c8b-0e58a020f2e5

United Nations General Assembly, 2015. Transforming Our World: The 2030 Agenda for Sustainable Development, https://doi.org/10.1007/s13398-014-0173-7.2

Wang, D., Brown, G., Zhong, G., Liu, Y., Mateo-Babiano, I., 2015. Factors influencing perceived access to urban parks: A comparative study of Brisbane (Australia) and Zhongshan (China). Habitat Int. 50, 335-346. https://doi.org/10.1016/j.habitatint.2015.08.032

WHO, 2012. Health indicators of sustainable cities in the context of the Rio+20 UN. Conference on Sustainable Development, Health Indicators of Sustainable Water in the Context of the Rio +20 UN Conference on Sustainable Development Key messages https://www.who.int/hia/green_economy/indicators_cities.pdf

Wolch, J.R., Byrne, J., Newell, J.P., 2014. Urban green space, public health, and environmental justice: The challenge of making cities "just green enough." Landsc. Urban Plan. https://doi.org/10.1016/j.landurbplan.2014.01.017

Wood, L., Hooper, P., Foster, S., Bull, F., 2017. Public green spaces and positive mental health - investigating the relationship between access, quantity and types of parks and mental wellbeing. Heal. Place 48, 63-71. https://doi.org/10.1016/j.healthplace.2017.09.002 